\title{
Predicting Traffic Dynamics with Driver Response Model for Proactive Variable Speed Limit Control Algorithm
}

\author{
Huixuan Ye, ${ }^{1}$ Lili Tu, ${ }^{1}$ and Jie Fang ${ }^{10}{ }^{1,2}$ \\ ${ }^{1}$ College of Civil of Engineering, Fuzhou University, Fuzhou, Fujian, China \\ ${ }^{2}$ University of Alberta, 3-019 NREF, Edmonton, AB, Canada T6G 2W2 \\ Correspondence should be addressed to Jie Fang; fangjie@fzu.edu.cn
}

Received 21 December 2017; Revised 24 February 2018; Accepted 22 March 2018; Published 6 May 2018

Academic Editor: Li Xu

Copyright (c) 2018 Huixuan Ye et al. This is an open access article distributed under the Creative Commons Attribution License, which permits unrestricted use, distribution, and reproduction in any medium, provided the original work is properly cited.

\begin{abstract}
Variable Speed Limit (VSL) control contributes to potential crash risk reduction by suggesting a suitable dynamic speed limit to achieve more stable and uniform traffic flow. In recent studies, researchers adopted macroscopic traffic flow models and perform prediction-based optimal VSL control. The response of drivers to the advised VSL is one of the most critical parameters in VSLcontrolled speed dynamics modeling, which significantly affects the accuracy of traffic state prediction as well as the control reliability and performance. Nevertheless, the variations of driver responses were not explicitly modeled. Thus, in this research, the authors proposed a dynamic driver response model to formulate how the drivers respond to the advised VSL during various traffic conditions. The model was established and calibrated using field data to quantitatively analyze the dynamics of drivers' desired speed regarding the advised VSL and current traffic state variables. A proactive VSL control algorithm incorporating the established driver response model was designed and implemented in field-data-based simulation study. The design proactive control algorithm modifies VSL in real-time according to the traffic state prediction results, aiming to reduce potential crash risks over the experiment site. By taking into account the real-time driver response variations, the VSL-controlled traffic state dynamics was more accurately predicted. The experimental results illustrated that the proposed control algorithm effectively reduces the crash probabilities in the traffic network.
\end{abstract}

\section{Introduction}

Active Transportation and Demand Management (ATDM) has become a well-known concept in the last decade. ATDM aims to mitigate daily recurrent traffic congestion with the support of real-time traffic data monitoring, processing, and control optimizing. It relies on fast emerging technology for traffic data capturing and information sharing in order to facilitate coordinated control and achieve optimal traffic conditions at the network level. Along with other active traffic control strategies, Variable Speed Limit (VSL) control is one of the most intensively studied control measures. The static speed limit posted on a highway section defines the highest driving speed allowed under prevailing traffic conditions. Nevertheless, such static speed limits may fail to suggest the most appropriate operating speed during certain extreme circumstances [1], including the following: a drop in capacity due to road construction, a change in road conditions due to severe weather (fog, snow storm, etc.), or high traffic demand generated by large events. Earlier studies reported that, by implementing VSL to enhance speed homogeneity, traffic conditions can be improved in terms of both safety [1-3] and mobility $[4,5]$.

Given that the nature of VSL control is to alter the operating speed of traffic flow, the response of drivers to the advised VSL is a critical factor in control performance. In reality, it is arbitrary to assume that traffic flow will follow the advised VSL value strictly. However, in the existing literature, other than not presenting the assumption of how the speed dynamic responds to VSL control, only a few studies in the past explicitly investigated how the speed dynamic responds to VSL control. Hellinga and Mandelzys [6] assumed four different possible levels of driver responses (low, medium, high, and very high) to the VSL and then examined the sensitivity of VSL's control performance over operational and safety benefits subject to these categories. Several other studies [7] 
adopted a similar approach to assume how certain percentages of drivers will respond to the advised VSL in simulation.

However, in the authors' opinion, the mechanism of driver responses to the VSL is a sophisticated system and it can hardly be represented accurately by simply assuming that the advised VSL will be complied with by a constant proportion of drivers. Instead, the response of flow speed to the VSL should vary according to the current traffic conditions. Moreover, such a dynamic response mechanism needs to be taken into consideration while anticipating future traffic states in prediction-based VSL control algorithms, such as $[3,8]$. Therefore, in this study, the authors propose to establish a macroscopic VSL response model, which describes the change in drivers' responses to the implemented VSL dynamically, according to the current traffic conditions. This relationship was formulated to associate drivers' desired speed dynamically with traffic state variables, such as current volume or occupancy. The model was conducted through a regression of field experiment data. For model evaluation, this paper adopts the prediction-based VSL control algorithm described in the authors' earlier article [3] and has further improved its traffic state prediction module.

The remainder of this paper is organized into sections: Section 2 is a brief literature review of existing VSL control strategies and related speed response modeling efforts; Section 3 presents the proposed driver response modeling approach and the corresponding VSL control algorithm; Section 4 presents a field-data-based VISSIM simulation study to reproduce real-world traffic conditions and implement the proposed control algorithm. The VSL-controlled network performance is compared with the baseline condition (uncontrolled case) to evaluate the efficiency of the proposed control algorithm. Lastly, Section 5 presents conclusions and future work.

\section{Literature Review}

Realizing the importance of drivers' responses to VSL control, previous studies have investigated the correlation between driver compliance and VSL control. Real-world field implementation was examined first in both Europe and North America to analyze the effects. In general, field implementation in Europe was enforced by police through means of cameras or other monitoring methods, which resulted in overall higher compliance compared to the advisory-only VSL control in North America.

One of the earliest VSL driver response studies in Europe was implemented on a 20 kilometer freeway section over A2 motorway near Amsterdam, Netherlands [9]. The study investigated drivers' responses to the installed VSL control system, which lowered the speed limit from over $90 \mathrm{~km} / \mathrm{h}$ to $70 \mathrm{~km} / \mathrm{h}$. Although the study reported that drivers follow the speed limit in general, the traffic flow dynamics under VSL control were not quantitatively analyzed to support the argument. Another study in Europe [10] was conducted in the UK on M25 motorway, which was automatically enforced by overhead cameras. Similarly, the study reported improved driver compliance with the VSL, although the increase was not quantified.
In North America, VSL field implementation was conducted in Washington [11]. In the Washington test, 25 VSL signs were installed on both directions of I-90 to reduce the traveling speed through construction zones. However, due to the existing traffic congestion, the study did not analyze the direct response to VSL control. The Minnesota study adopted a two-stage speed reduction scheme at work zone bottlenecks along I-494 in Twin Cities. The field data demonstrated that the average speed difference (1-minute interval) was reduced by 25 to 35 percent. It also reported that drivers' compliance was better when there was a larger gap between the current speed and advised speed limit. Another speed response test was conducted on a six-mile work zone section over I-80 near Wanship, Utah. The results showed that traffic flow followed the VSL closely and that there was no statistically significant difference in between. Chang et al. worked on integrating variable speed control and travel time information for alleviating day-to-day recurrent congestion on a highway corridor [12]. The VSL control and travel time information was updated based on real-time traffic condition collected by licenseplate-recognition system.

More literature on this topic can be found among simulation-based studies. One reprehensive study is Hellinga and Mandelzys's work [6] in 2011, which tested server compliance levels to evaluate the sensitivity of the safety and operational impacts of VSL on driver compliance in PARAMICS. Four levels of driver response (low, medium, high, and very high) to the VSL were assumed and used to testify the impact of the response on control performance. They reported that safety is positively correlated with driver compliance, while the analysis of travel time went the other way.

The study mentioned above implemented VSL control based on logics using predefined thresholds, such as a decision tree [6]. Another approach to implementing VSL control is to adopt a macroscopic traffic flow model and perform prediction-based optimal control. Hegyi et al.s work $[8,13,14]$ is among the earliest studies on this approach. The author adopted the Model Predictive Control (MPC) framework to facilitate VSL control based on the prediction results from an extended version of the famous macroscopic traffic flow model: METANET. The control vector (advised VSL) was introduced into the original model to replace the speed expectation, and a driver compliance modifier (as a percentage) was attached to the control vector.

Carlson et al. [15] used a similar framework which adopts a macroscopic traffic flow model (modified METANET model) and performed prediction-based optimal control. The impact of driver compliance was not explicitly included in the prediction stage, and thus it was not clear how it affected the control performance.

The work by Hadiuzzaman et al. [7] further extended the study regarding the compliance level using MPC-based control. Multiple compliance levels were assumed for VSL control and the control benefits were measured. It was reported that VSL control has benefits in terms of both mobility and safety aspects over most compliance level assumptions, but the nature of the dynamic driver response is still not clear. Yang et al's recent work $[16,17]$ proposed to incorporate Kalman filter in the VSL control algorithm design, which was used 
to surpass the uncertainty brought into the system by the driver compliance. The predicted result was used to optimize VSL targeting at travel time minimization and speed variance minimization.

In summary, there are only a few studies available to examine the critical relationship between driver response and VSL control. The existing literature either neglects this critical effect [15] or addresses it by assuming that a fixed proportion of drivers will follow the advised VSL $[6-8,18,19]$, regardless of changing traffic state variables. This has two major drawbacks: (1) Assuming one (or several) fixed driver response proportion is not necessarily accurate for all scenarios. In reality, the drivers' response (change of desired speed) to the VSL will change along with current traffic conditions and the corresponding VSL. For example, during a congested period, drivers might follow a VSL of $60 \mathrm{~km} / \mathrm{h}$ much better than during free-flow conditions. (2) As a sophisticated and critical effect, the drivers' response was not considered before optimizing the control. That is to say, the anticipated control performance provided by the existing prediction-based optimal control algorithms did not quantitatively measure the impact of driver compliance to the VSL. A model of drivers' responses must be included in the macroscopic traffic flow model for predicting the future traffic state after control effort.

Therefore, in this paper, the authors propose to analyze the mechanism of drivers' responses to the advised VSL based on the field data and establish a model to describe how traffic flow dynamics change according to different traffic states and the VSL control signal. Furthermore, this model has been introduced to improve a prediction-based VSL control algorithm by considering the impact of drivers' responses and providing more precise evidence for traffic state prediction before control optimization and implementation.

\section{Prediction-Based VSL Control}

In order to evaluate the performance of the proposed driver response model, an enhanced prediction-based active traffic control algorithm previously proposed by the authors in [3, 20] was adopted in this study. For the readers' convenience, here the authors briefly introduce the basic concept of the control algorithm and where the driver response model proposed in this paper fits in.

As indicated in Figure 1, the controller of the designated algorithm includes three major steps: traffic state predicting, control performance assessment, and optimization. The adopted control algorithm optimizes the control plan based on the current traffic conditions (traffic state variables). The traffic state predictions are estimated using the combined application of the macroscopic traffic flow model and the driver response model. Considering the drivers' dynamic response to the control inputs, these two models incorporate both the current traffic state $x(k)$ and the control plan $u(k)$ to predict the traffic state at the consecutive time step. The estimated traffic states and their associated control inputs are then evaluated by the system assessment model to select the optimal control input $u^{*}(k)$ with the best system performance. After applying the selected control input $u^{*}(k)$ to the traffic network, the updated traffic state variables are sent to the controller again to optimize the control input for the next time step.

In addition to the driver response model, a macroscopic traffic flow model is needed for predicting traffic states. A classic macroscopic traffic flow model, such as METANET, divides a freeway into discrete links to analyze their spatialtemporal aggregated characteristics at divided time steps. Lu et al.s model [18] is a modified version of the METANET model: the fundamental diagram assumption in the original METANET model is replaced by introducing the VSL control variable VSL $\left(u_{m}(k)\right)$ directly into the model.

Lu's model is composed of the following equations:

$$
\begin{aligned}
\rho_{m}(k & +1) \\
= & \rho_{m}(k) \\
& +\frac{T}{L_{m} \lambda_{m}}\left[q_{m-1}(k)-q_{m}(k)+q_{m, \text { on }}-q_{m, \mathrm{off}}\right] \\
v_{m}(k+1) & v_{m}(k)+\frac{T}{\tau}\left(u_{m}(k)-v_{m}(k)\right) \\
& +\frac{T}{L_{m}} v_{m}(k)\left(v_{m-1}(k)-v_{m}(k)\right) \\
& -\frac{\mu T}{\tau L_{m}}\left(\frac{\rho_{m+1}(k)-\rho_{m}(k)}{\rho_{m}(k)+\kappa}\right),
\end{aligned}
$$

where $m$ represents the index of the links, while $k$ represents the index of the time steps; $v_{m}(k), q_{m}(k)$, and $\rho_{m}(k)$ are the speed, volume, and density of link $m$ at the current time step $k ; T$ is the length of the time step; $L_{m}$ and $\lambda_{m}$ are the length and number of lanes in link $m ; q_{m \text {,on }}$ and $q_{m \text {,off }}$ are the volume at the on-/off-ramp $m ; u_{m}(k)$ is the advised VSL value at link $m ; \tau, \mu$, and $\kappa$ are the model parameters.

Equation (1) is the flow conservation equation. This equation expresses the flow conservation and calculation to measure the density at the consecutive time step. On the other hand, (2) interprets the mechanism of anticipating the speed dynamics. The velocity in the forthcoming time step was predicted by modifying the current link speed with three different terms: the relaxation term, convection term, and anticipation term. Among these three terms, the relaxation term measures the impact of the drivers' desired speed on the traffic flow. In the original METANET model, this term assumes that drivers will tend to accelerate/decelerate towards a desired speed (which is the value from the fundamental diagram assumption). In VSL-controlled environment, while the control signal (advised VSL) is missing, the original speeddensity relationship assumption (FD diagram) proposed in METANET does not necessarily represent the driver response accurately.

$\mathrm{Lu}$ et al's and a couple of other later research works $[3,7,18]$ replaced the desired speed directly with the advised VSL value $u_{m}(k)$, assuming that traffic will follow the advised VSL value strictly. As explained before, to more accurately reflect the real-world situation, this anticipation term should 


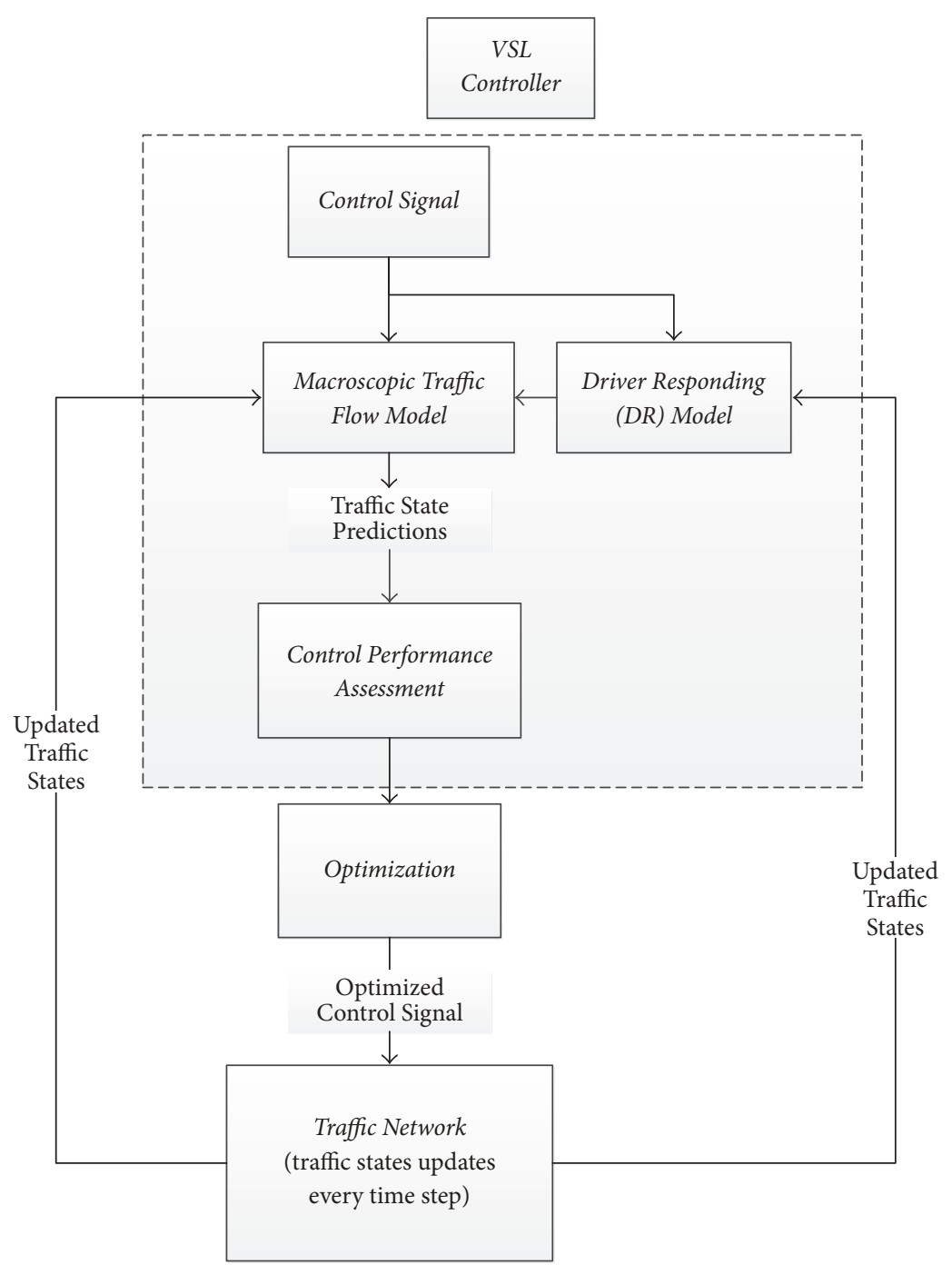

FIgURE 1: Established driver response (DR) model and adopted control algorithm.

be qualified to treat driver responses differently under different traffic conditions. Thus, in this study, the authors use the proposed driver response model to modify this term. The modified speed dynamic equation is expressed in the equation below:

$$
\begin{aligned}
v_{m}(k+1)= & v_{m}(k)+\frac{T}{\tau}\left(\operatorname{DR}(k+1)-v_{m}(k)\right) \\
& +\frac{T}{L_{m}} v_{m}(k)\left(v_{m-1}(k)-v_{m}(k)\right) \\
& -\frac{\mu T}{\tau L_{m}}\left(\frac{\rho_{m+1}(k)-\rho_{m}(k)}{\rho_{m}(k)+\kappa}\right)
\end{aligned}
$$

Basically, this modified model adopted the proposed driver response model $(\mathrm{DR}(k+1))$ to replace the control variable in the relaxation term. By introducing this model, the drivers' desired speed does not simply equal the advised VSL (as in the original model). Instead, the drivers' responses to the advised VSL under current traffic conditions are formulated. This modification will further improve the accuracy of traffic state prediction under various traffic conditions.

\subsection{Modeling the Driver Response Mechanism}

3.1.1. Model Formulation. To more accurately reflect the dynamic driver response mechanism in VSL control, the regression technique was employed to model the relationship among traffic status, control signal (advised VSL value), and drivers' response (the drivers' desired speed). It should be noted that the field-collected flow speed can only be considered as the desired speed when traffic is uncongested. Otherwise, the flow speed is restrained by the traffic conditions and hardly reflects the true desired speed associated with the current VSL value. Moreover, it has been proven that VSL mainly works to improve traffic operations by postponing the traffic breakdown [12]. After the traffic breakdown happens, drivers fall into a stop-and-go situation other than following the advised VSL. Thus, to design a drive response model that 
works for VSL prediction and control optimization purpose, the main focus of the model should be investigating the data above the jammed region, which is the function of VSL control to lower the driving speed beforehand and postpone traffic breakdown. Therefore, in this work, the authors mainly focused on examining the scenarios with relatively high VSL values $(60 \mathrm{~km} / \mathrm{h}-80 \mathrm{~km} / \mathrm{h})$ to avoid having noise in the observed data.

During the test, a total of five variables were considered to establish the model, as expressed in

$$
\begin{aligned}
\operatorname{DR} & (k+1) \\
\quad= & R\left(u(k), v(k), v^{\wedge}(k), q(k), \rho(k), \rho(k+1)\right) .
\end{aligned}
$$

And four model formulations were tested to examine which better represents this dynamic driver response mechanism:

$$
\begin{aligned}
& \mathrm{DR}(k+1)=C+a x_{1}+b x_{2}+\cdots+z x_{p} \\
& \mathrm{DR}(k+1)=a x_{1}+b x_{2}+\cdots+z x_{p} \\
& \mathrm{DR}(k+1)=C+a\left(x_{1}\right)^{x}+b\left(x_{2}\right)^{y}+\cdots+z\left(x_{p}\right)^{z} \\
& \operatorname{DR}(k+1)=a\left(x_{1}\right)^{x}+b\left(x_{2}\right)^{y}+\cdots+z\left(x_{p}\right)^{z},
\end{aligned}
$$

where DR represents the driver responses. In order to incorporate the driver response into a macroscopic traffic flow model, it was represented by the averaged individual vehicle driving speed.

$k$ represents the index of time steps; $u(k)$ is the advised VSL value; $v(k)$ is the flow speed at time step $k ; v^{\wedge}(k)$ is the difference between current speed $v(k)$ and advised VSL $u(k)$; $q(k)$ is the volume at time step $k ; \rho(k)$ and $\rho(k+1)$ are the observed density at time step $k$ and predicted density at time step $k+1$, respectively.

3.1.2. Data Preparation and Processing. The data for modeling the driver responses were collected at a major connector highway installed with DSDSs (Dynamic Speed Display Signs) in Edmonton, Alberta, Canada. See Figure 2 for the demonstration of the experiment site.

The demonstrated highway corridor has a static speed limit of 80 kilometers per hour posted. The DSDSs were installed with sensors at the four locations (sites 1-4) as illustrated on the map to record the drivers' dynamic response to the advised speed limit: individual speed, volume, and occupancy (aggregated by 60-second interval). Among them, sites 2 and 4 were considered as advanced warning for drivers to decelerate from the original speed limit, and traffic state variables were collected at sites 2 and 4 to analyze their responses. A warring sign was placed about $400 \mathrm{~m}$ beforehand of the lowered speed limit sign. The whole speed decreasing segment is about $1.6 \mathrm{~km}$, in which the driver will need to break from 80 to $60 \mathrm{kph}$. The distance among the signs was set to be consistent with the length of road segment in the field validation test. In the field, it is observed that most drivers were able to adjust their speed within the setup region, since its length is similar to the typical advance warning sign of offramp and that on the freeway.

Traffic flow characteristics and vehicle classification data were collected for 63 continuous days with DSDS in use and 38 days without. The dataset obtained while the DSDSs were on was used. 28 days of data were used for model parameter calibration and 27 days of data were used for model validation (both with and without DSDS in use). Among the selected variables, segment speed and speed limit were directly measured, while segment density required postprocessing. The simplified equation below was used for calculating the segment density based on the available data (occupancy):

$$
\begin{aligned}
& \rho=\text { occ. } \times \frac{52.8}{(L v+L s)} ; \\
& L v=N_{\text {bin } 1} L_{\text {bin } 1}+N_{\text {bin } 2} L_{\text {bin } 2}+\cdots+N_{\text {binn }} L_{\text {binn }} L s=0.5 \mathrm{ft},
\end{aligned}
$$

where occ. is the occupancy data collected by the sensor; $\rho$ is the segment density; $L v$ is the averaged vehicle length during one data collection interval; $N_{\text {bini }}$ is the vehicle count within one interval for classification $i ; L_{\text {bini }}$ is the average vehicle length for classification $i$, which is the median value of the upper bound and lower bound of each classification.

3.1.3. Driver Response Model Calibration and Validation. In order to establish the model and calibrate the model parameters, both linear and nonlinear regression techniques were performed to analyze the aforementioned model formulations (I) (IV) and the results are demonstrated in Table 1.

According to the regression analysis results, model formulation (II) outperformed the other three model formulations with the highest adjusted $R$ square value of 0.92 . Therefore it was selected as the formulation of the proposed model. One underlying reason of this selection is in order to exclude the disturbances from congestion (enforced speed reductions); this research analyzed the traffic data out of the jammed region. Thus, the driver response is more introductive but nonlinear as in the breakdown state. As mentioned before, the driver responses among this transition region between free-flow and congestion should be focused on the VSL control study in order to benefit the road users.

Furthermore, a significance level of 0.05 was selected as the criterion for including a particular variable in the final model, which excluded two candidate variables: the volume rate $q(k)$ and density $\rho(k)$. The advised VSL value $u(k)$, current speed $v(k)$, the predicted segment density $\rho(k+1)$ according to the flow changes, and $v^{\wedge}(k)$ the difference between current speed and advised speed were found to be statistically significant in the model. Since $u(k)$ and $v(k)$ are correlated to $v^{\wedge}(k)$, the one with lower significance level $(v(k)$, significance level $=0.03$ ) was removed and the model was recalibrated. The final model and model estimates were shown in Table 1(b).

As shown in Table 1(b) of parameter estimation results, the magnitude of the parameter indicates that the speed limit has a larger impact than the current traveling speed. The estimated coefficient of density is negative, which suggests that drivers tend to lower their speed when the traffic is dense. Figure 3 shows the detailed model validation results. 

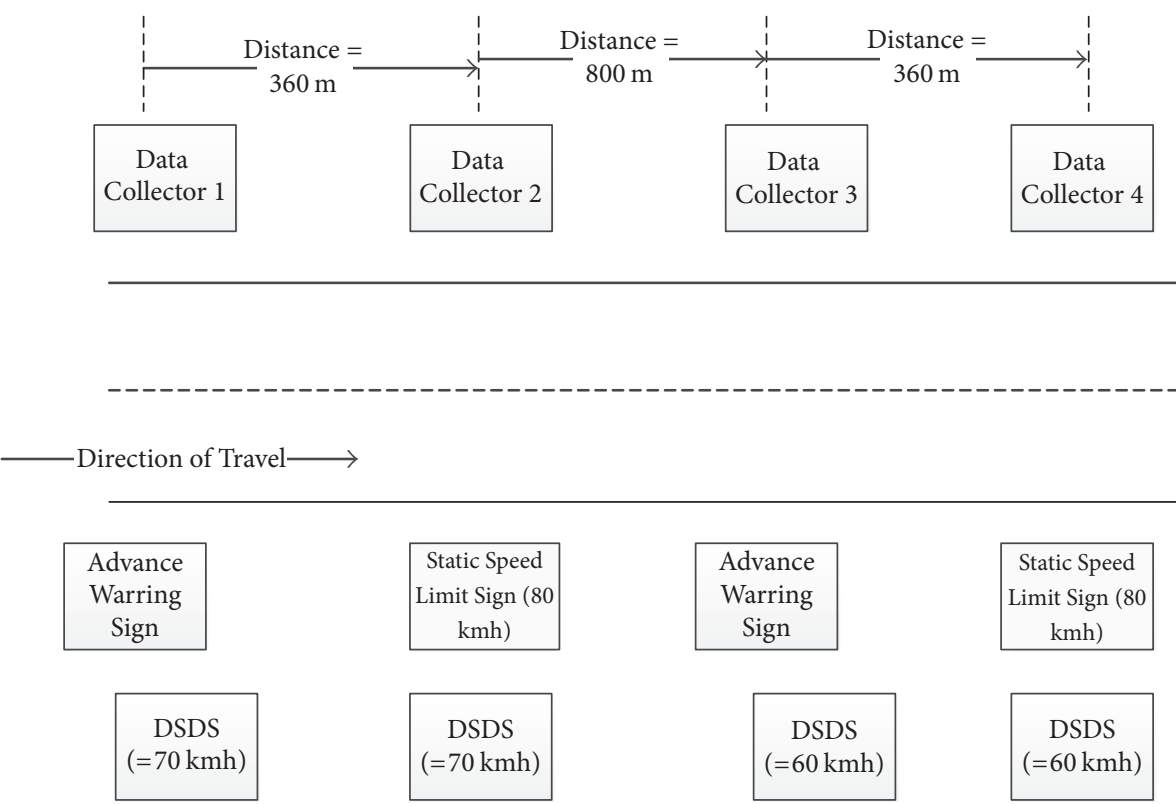

FIgURE 2: Experiment site layout in Edmonton, AB, Canada.

TABLE 1

(a) Statistics of examined model formulations

\begin{tabular}{lcccc}
\hline Variable & Model formulation (I) & Model formulation (II) & Model formulation (III) & Model formulation (IV) \\
\hline$R$ square & 0.81 & 0.92 & 0.71 & 0.84 \\
Adopted variable & Con, $u(k), v(k), v^{\wedge}(k), \rho(k+1)$ & $u(k), v^{\wedge}(k), \rho(k+1)$ & Con, $u(k), v(k), v^{\wedge}(k), \rho(k+1)$ & $u(k), v^{\wedge}(k), \rho(k+1)$ \\
\hline
\end{tabular}

(b) Parameter estimates for the adopted model (formulation (II))

\begin{tabular}{|c|c|c|c|c|}
\hline Variable & Parameter estimate & Standard error & Significance & $R$ square \\
\hline$u(k)$ & 0.659 & 0.016 & 0.01 & \\
\hline$v^{\wedge}(k)$ & 0.474 & 0.008 & 0.01 & 0.92 \\
\hline$\rho(k+1)$ & -0.577 & 0.038 & 0.03 & \\
\hline
\end{tabular}

For model validation, the proposed model was adopted to predict the drivers' responses in the validation dataset. As demonstrated in Figure 3, the model-predicted value matches the field data closely (mean error $<1 \mathrm{~km} / \mathrm{h}$, std $=6.16 \mathrm{~km} / \mathrm{h}$ ). Also, there is no significant bias observed since the median and average drop within a $0-4 \mathrm{~km} / \mathrm{h}$ range. Therefore, it is concluded that the proposed model can be used to formulate the dynamic driver response mechanism. Therefore the following calibrated driver response model DR (6) can be substitute into the speed dynamics model (3) for real-time VSLcontrolled speed predictions.

$$
\begin{aligned}
\operatorname{DR}(k+1)= & 0.659 u(k)+0.474 v^{\wedge}(k) \\
& -0.577 \rho(k+1) .
\end{aligned}
$$

3.2. VSL Control Optimization. As shown in the control algorithm (Figure 1), the optimization module in the adopted VSL control algorithm takes the evaluation results from the network performance assessment module to determine the optimized VSL control input. Although the proposed traffic prediction model process and updates the prediction result at 20-second interval, in practice, it is unrealistic for drivers to adjust their desire in such high frequency. Altering the advised VSL by such short interval will confuse the drivers and therefore encouraging speed violations instead. Thus, in the control algorithm, the VSL control signal was optimized every 5 minutes according to predicted network safety performance. The network performance is determined by a precursor collision prediction model the authors presented in their previous research $[3,5]$ and the following equation:

$$
\begin{aligned}
& P(u(k)) \\
& \quad=\frac{\exp \left(\operatorname{con}+\alpha \mathrm{SV}_{2}+\beta \log \mathrm{AO}_{1}+\gamma \log \mathrm{SS}_{2}\right)}{1+\exp \left(\operatorname{con}+\alpha \mathrm{SV}_{2}+\beta \log \mathrm{AO}_{1}+\gamma \log \mathrm{SS}_{2}\right)},
\end{aligned}
$$

where $\mathrm{SV}_{2}$ is the standard deviation of volume in the last 5-10 minutes;

$\mathrm{AO}_{1}$ is the average occupancy in the last $0-5$ minutes before; 


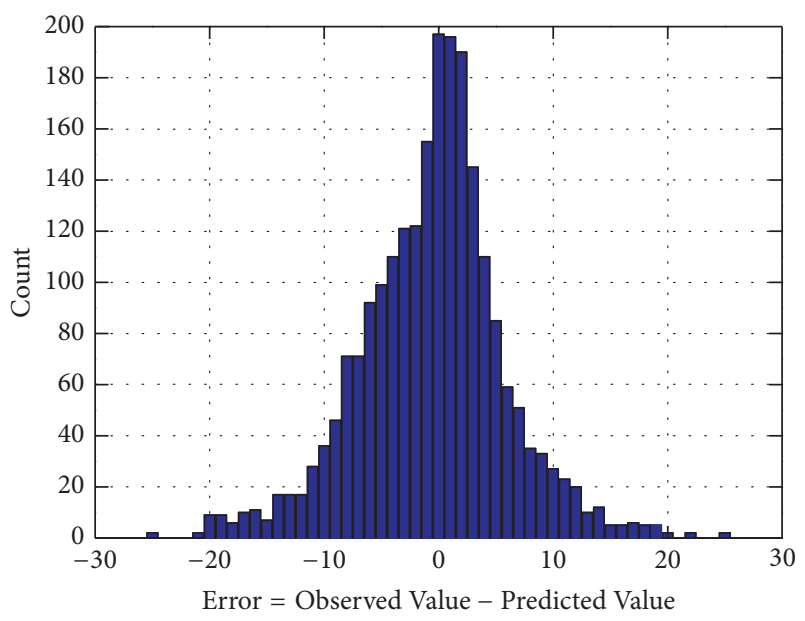

FIGURE 3: Validation results of proposed driver response model (one week of data, error units: $\mathrm{km} / \mathrm{h}$ ).

$\mathrm{SS}_{2}$ is the standard deviation of speed 5-10 minutes prior to the incident;

Con, $\alpha, \beta$, and $\gamma$ are the model parameters to be calibrated.

Equation (6) was established through the logistic regression technique to identify the critical traffic state variables for modeling the probability of traffic incidents. For more details of how the crash probability was established, system constraints, and control plan optimization techniques of the control algorithm, the authors refer to their previous study $[3,5]$.

The optimization aims to minimize the overall system safety risk, which is a problem of minimizing the objective function $J$ over the examined prediction horizon $N_{p}$ (the time window of the predicted traffic states, set at 10 minutes in this study).

$$
J(u(k))=\sum_{j=1}^{N_{p}-1} \sum_{i=1}^{M} P_{i, j}\left(u_{i, j}(k)\right)
$$

The objective function in (8) measures the summation of measured incident probability over all of the $m$ analyzed links within the prediction horizon. The collision probabilities were evaluated at every link and at all time steps within the prediction horizon $N_{p}$, separately for each feasible control input. The average overall collision probability was then used to determine the optimal VSL control input.

The authors adopted a simple SQP (Sequence Quadratic Program) to solve the control optimization problem. In the selected experiment site, the adopted SQP method was able to converge the optimization within minutes, which makes it suitable for online application. The computation complexity may vary depending on the required optimization accuracy and the number of control variables. For more discussion regarding the optimization technique, the authors would refer to another article that works on solving complex multiobjective optimization problem in active freeway control [21].

\section{Evaluation and Results Analysis}

4.1. Experimental Design. To assess the efficiency of using the proposed model in the prediction-based VSL control algorithm, a field-data-based simulation study was conducted. A local freeway corridor allocated in the same region as the model calibration site was chosen for evaluation. The selected corridor is the northbound segment of Whitemud Drive (WMD), which is a major highway that serves as the inner ring road of Edmonton, Alberta, Canada (Figure 4).

According to the local Department of Transportation (DOT), the WMD corridor carries heavy commuter trips of more than 40,000 in AADT (Annual Average Daily Traffic). Over 30 loop detectors installed along the corridor monitor the real-time traffic conditions along the corridor at 60 second intervals. The detector report shows that the corridor suffers from daily recurrent congestion during peak hours for most weekdays; the flow speed is lower than $50 \mathrm{~km} / \mathrm{h}$ for more than $70 \%$ of the workday peak hours. For evaluation, the selected corridor was projected into the microscopic traffic simulation tool VISSIM with a complete morning peak hour demand profile (collected from the field by the loops, 6:00-9:00 AM). To model the network, the selected experiment site was divided into 13 links (as shown in Figure 4). The underlying principle of segmentation is that, within each link, traffic characteristics should be consistent, for example, the same lane number and speed limit. Furthermore, for each link, it should possess at least one loop detection station, from which the 20-second interval loop detector data were processed to represent the real-time traffic condition on that link. The simulation platform was calibrated by these corresponding field-collected loop detector data.

In the selected corridor, two bottleneck locations were identified, and thus four VSL signs were placed before and after each of the identified bottlenecks and optimized by the proposed VSL algorithm with the enhanced driver response model. In order to accurately reproduce the driver behavior during VSL control, the driver behavior profile on the site was calibrated for different VSL values according to the aforementioned DSDS data. Since the DSDS data and the WMD corridor are in the same area (6-8 km apart) and both carry mostly commuting traffic, the authors assumed that the characteristics of their driver responses to the VSL control were similar. With such calibration, the simulated traffic flow will respond to the VSL differently instead of receiving the same traffic flow characteristics for all VSL values; this procedure will make the simulation work more realistic and close to the actual field situation.

4.2. Result Analysis. To appraise the proposed VSL control algorithm, the safety performance of the selected corridor without VSL control was studied as the baseline condition and compared with the VSL-controlled scenario. To quantitatively perform the evaluation, the collision probability $(\mathrm{CP})$ was taken to measure the network-wide safety performance. Using the adopted network safety performance model (7), CP was measured for each link at each time step and the average overall CP was recorded for comparison. It should be noted that the first half-hour of the study period was considered 


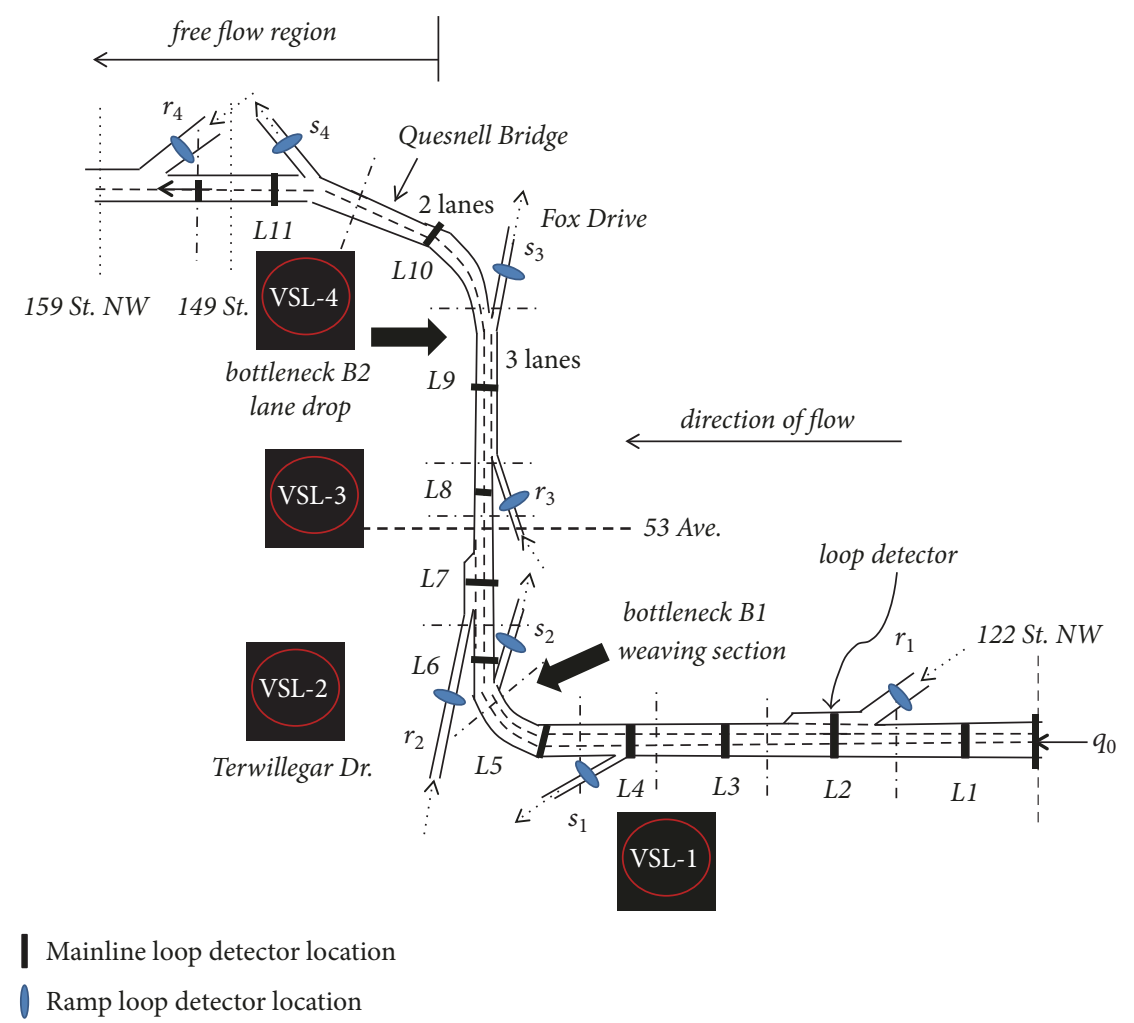

FIGURE 4: Layout of the experimental site.

as the warm-up period and excluded from the comparisons; links 1 and 11 were outside of the VSL-controlled area, so they were excluded from the comparison as well. Figure 5 and Table 2 illustrate the detailed comparison results.

First of all, by examining the average CP across the entire study area, it can be concluded that the implemented VSL control has an overall positive impact on the experiment corridor. The average CP in the VSL-controlled scenario was reduced by $32 \%$, relatively (from 19\% to 13\%). Table 2 further splits the experiment duration into five 30-minute segments. The comparison results show that, for the first hour, there was no major difference between the two tested scenarios. The reason is, for that period, that there was no heavy traffic demand in the network and the safety performance of the corridor was fine (CP less than 10\%). Thus, the control algorithm was not active to restrict the traffic flow. Nevertheless, during the congestion period (8:00-9:00 AM) where the CP went over $25 \%$ due to the oversaturated demand and congested traffic flow (high density), the corridor CP level was effectively controlled by implementing the proposed VSL system. The overall CP of the corridor was significantly reduced by over $35 \%$, relatively.

Secondly, for individual link evaluation results, all the examined links show a positive result by reporting effectively reduced crash potential. Among the tested links, the most significant CP reduction was observed at link 6 (49\%, relatively). Link 6 is one of the major identified bottlenecks in the evaluated corridor. Thus, here the authors take link 6 as an example to further demonstrate how the implemented VSL control works.
Figure 6 illustrates the change in CP for link 6 with and without VSL control. The demonstrated link suffers serious congestion without VSL (pale dash line). The speed profile drops to $20 \mathrm{~km} / \mathrm{h}$ after the peak demand hits the bottleneck around 7:30 AM and does not recover until the end of the experiment (approximately 8:40 AM).On the other hand, relying on the proposed macroscopic traffic flow model and driver response model, the VSL control algorithm successfully predicted the oversaturated demand and forthcoming traffic breakdown in advance. As a response, the control algorithm lowered the speed limit gradually before the traffic breakdown at approximately 7:15 AM. This scheme helped the traffic stream to slow down step by step, which brought down the crash risk caused by the abrupt traffic breakdown. Furthermore, the control strategy effectively mitigated the scale of the congestion (flow speed recovered at about 8:00 AM instead of after 8:40 AM), which also lowered the CP by reducing the length of the high density region. The observed $\mathrm{CP}$ reduction is the joint effort of corridor-wide coordinated multilink control, and it is consistent with the corridor-level CP reduction observed in Table 2 .

Another interesting fact is the experiment reflects the dynamic response mechanism of the drivers to VSL: at the very outset, when the control algorithm lowered the speed limit before traffic breakdown (7:10 to 7:25 AM), the drivers did not follow the advised VSL considerably. Some even disobeyed the advised speed limit. However, later on when traffic was more congested (7:30 to 8:00 AM), the drivers appeared to follow the VSL more strictly or even drive lower than the advised value. This observation proves that the 
TABLE 2: Comparison of crash probability with and without proposed VSL control.

\begin{tabular}{|c|c|c|c|c|c|c|}
\hline Crash probability (\%) & 6:30-7:00 AM & 7:00-7:30 AM & 7:30-8:00 AM & 8:00-8:30 AM & 8:30-9:00 AM & Overall \\
\hline Uncontrolled & 0.6 & 8.6 & 27.4 & 29.0 & 25.2 & 19.8 \\
\hline VSL control & 0.6 & 8.3 & 23.1 & 18.6 & 13.3 & 13.4 \\
\hline $\mathrm{CP}$ reduction (absolute) & 0 & 0.3 & 4.3 & 10.4 & 11.9 & 6.4 \\
\hline $\mathrm{CP}$ reduction (relative) & 0.0 & 3.4 & 15.7 & 35.7 & 47.1 & 32.3 \\
\hline
\end{tabular}

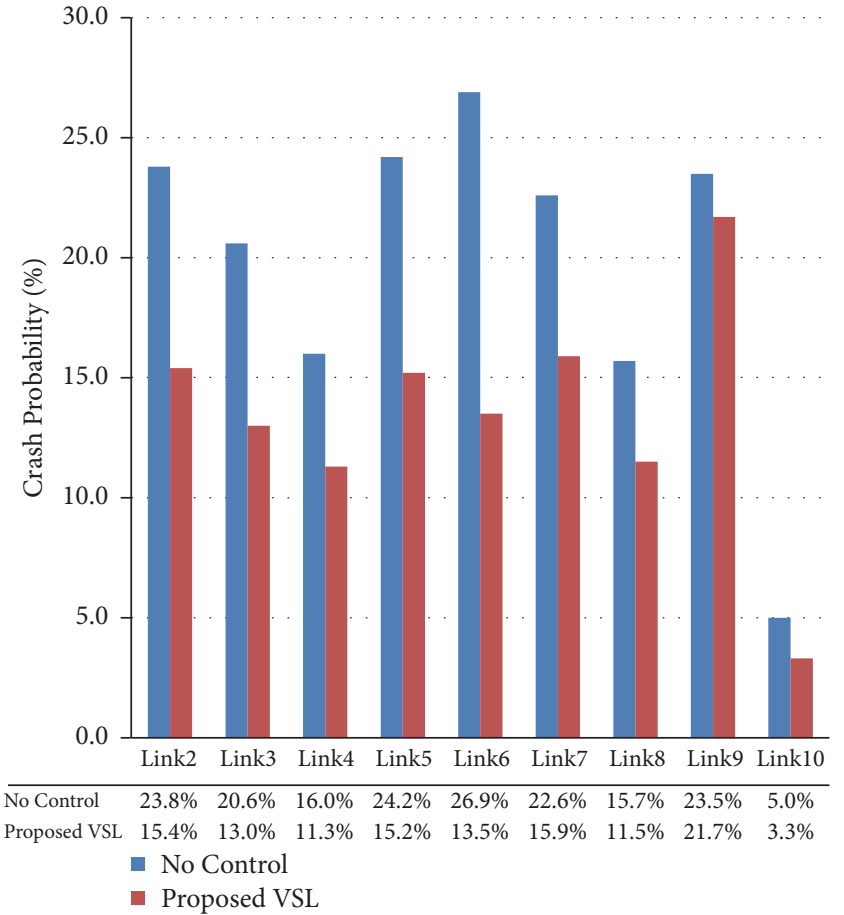

FIGURE 5: Comparison of crash probability profile with and without proposed VSL control.

conducted experiment and proposed control algorithm capture the nature of the dynamic response mechanism and provide a more realistic control practice. The authors give credit to the field data calibrated driver response model and simulations.

\section{Conclusions and Future Work}

In this research study, the authors proposed a dynamic VSL driver response modeling approach. Taking the current VSL value and traffic state variables as the independent variables, the model formulates the critical factor in safety oriented VSL control: the drivers' sophisticated response to various advised VSLs. The analysis of field data revealed how the current flow speed, density, and VSL value were associated with the desired speed. After calibration, the established model showed a close match to the field observation in the model validation process.

Furthermore, the proposed model was adopted to incorporate dynamic driver response mechanism modeling into a prediction-based optimal VSL control algorithm. For model evaluation, this control algorithm was used to implement VSL control over a congested highway corridor. The experiment

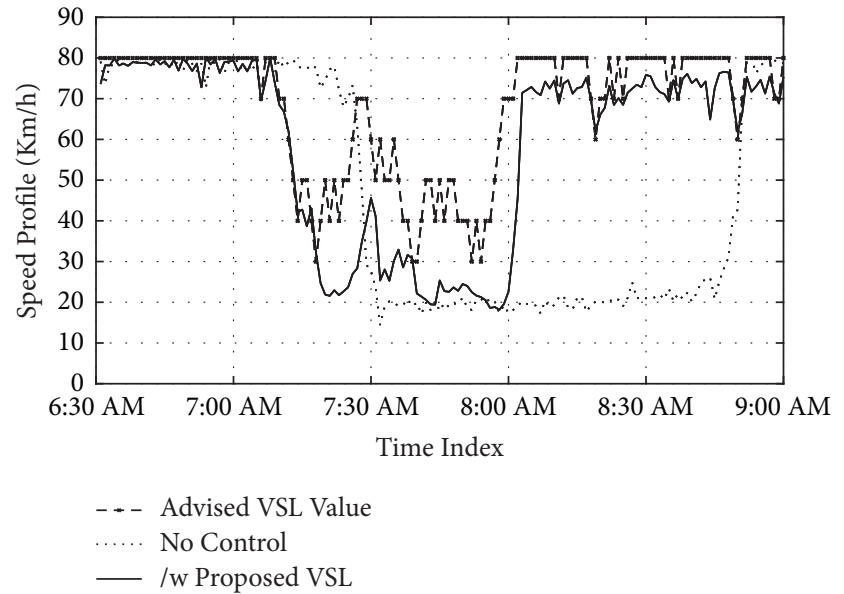

FIGURE 6: Speed profile of link 6 during experiment.

result demonstrated that the implemented VSL control has an overall positive impact on the experiment corridor. The average CP in the VSL-controlled scenario was reduced by $32 \%$, relatively. By the exertion of the proposed macroscopic traffic flow model and driver response model, the VSL control algorithm successfully predicted the oversaturated demand and forthcoming traffic breakdown in advance. Thus, the control algorithm was able to reduce the probability of crashes, by (1) forcing the traffic flow to slow down gradually beforehand and (2) mitigating the scale of the congestion period. Moreover, the conducted experiment and proposed control algorithm were able to capture the nature of the dynamic driver response mechanism and produce a more realistic control practice.

For future studies, the authors plan to evaluate the proposed VSL control algorithm in real-world applications. The WMD corridor evaluated in this paper has been equipped with proposed VSL signs and the dynamic control system. The authors look forward to reporting the field experiment results.

\section{Conflicts of Interest}

The authors declare that there are no conflicts of interest regarding the publication of this paper.

\section{Acknowledgments}

This work was jointly supported by the Fujian Science and Technology Department and Fujian Provincial Education 
Department, which provided support for the projects entitled "Traffic Flow Prediction and Evaluation Method of Urban Expressway Under Intelligent Traffic Control Environment" (Award no. 2015J05100) and "Multi-Objective Online Comprehensive Evaluation Method of Network Operation State" (Award no. JA15081), respectively.

\section{References}

[1] M. Abdel-Aty, J. Dilmore, and L. Hsia, "Applying variable speed limits and the potential for crash migration," Transportation Research Record: Journal of the Transportation Research Board, vol. 1953, pp. 21-30, 2006.

[2] P. Rämä, "Effects of weather-controlled variable speed limits and warning signs on driver behavior," Transportation Research Record: Journal of the Transportation Research Board, vol. 1689, pp. 53-59, 1999.

[3] J. Fang, M. Hadiuzzaman, A. Karim, Y. Luo, and T. Z. Qiu, "Variable speed limit control strategy with collision probability assessments based on traffic state prediction," Transportation Research Record, vol. 2435, pp. 11-18, 2014.

[4] M. Abdel-Aty, R. J. Cunningham, V. V. Gayah, and L. Hsia, "Dynamic variable speed limit strategies for real-time crash risk reduction on freeways," Transportation Research Record, vol. 2078, no. 1, pp. 108-116, 2008.

[5] M. Tazul Islam, M. Hadiuzzaman, J. Fang, T. Qiu, and K. ElBasyouny, "Assessing mobility and safety impacts of a variable speed limit control strategy," Transportation Research Record, no. 2364, pp. 1-11, 2013.

[6] B. Hellinga and M. Mandelzys, "Impact of driver compliance on the safety and operational impacts of freeway variable speed limit systems," Journal of Transportation Engineering, vol. 137, no. 4, pp. 260-268, 2011.

[7] M. Hadiuzzaman, J. Fang, M. A. Karim, Y. Luo, and T. Z. Qiu, "Modeling driver compliance to VSL and quantifying impacts of compliance levels and control strategy on mobility and safety," Journal of Transportation Engineering, vol. 141, no. 12, Article ID 04015028, 2015.

[8] A. Hegyi, M. Burger, B. De Schutter, J. Hellendoorn, and T. J. J. Van Den Boom, "Towards a practical application of model predictive control to suppress shock waves on freeways," in Proceedings of the 2007 9th European Control Conference, ECC 2007, pp. 1764-1771, July 2007.

[9] E. van den Hoogen and S. Smulders, "Control by variable speed signs: results of the Dutch experiment," in Proceedings of the Seventh International Conference on Road Traffic Monitoring and Control, pp. 145-149, London, UK, 1994.

[10] UK Highways Agency, M25 Controlled Motorway Summary Report, Highways Agency Publications Group, Bristol, UK, 2006.

[11] Washington State DOT, "I-90-Two-way transit and HOV operations-Variable speed limit signs," 2009, http://www.wsdot .wa.gov/Projects/I90/TwoWayTransit/vsl.html.

[12] G.-L. Chang, S. Y. Park, and J. Paracha, "Intelligent transportation system field demonstration: integration of variable speed limit control and travel time estimation for a recurrently congested highway," Transportation Research Record, no. 2243, pp. 55-66, 2011.

[13] A. Hegyi and S. P. Hoogendoorn, "Dynamic speed limit control to resolve shock waves on freeways - Field test results of the SPECIALIST algorithm," in Proceedings of the 13th International IEEE Conference on Intelligent Transportation Systems, ITSC 2010, pp. 519-524, Funchal, Portugal, September 2010.
[14] A. Hegyi, Model Predictive Control for Integrating Traffic Control Measures, Netherlands TRAIL Research School, 2004.

[15] R. C. Carlson, I. Papamichail, M. Papageorgiou, and A. Messmer, "Optimal mainstream traffic flow control of large-scale motorway networks," Transportation Research Part C: Emerging Technologies, vol. 18, no. 2, pp. 193-212, 2010.

[16] X. Yang, Y. Lu, and G.-L. Chang, "Exploratory analysis of an optimal variable speed control system for a recurrently congested freeway bottleneck," Journal of Advanced Transportation, vol. 49, no. 2, pp. 195-209, 2015.

[17] X. Yang and Y. C. Lu, "Development of optimal variable speed limit control system for freeway work zone operations," Transportation Research Board Meeting, vol. 94, pp. 149-160, 2014.

[18] X.-Y. Lu, P. Varaiya, R. Horowitz, D. Su, and S. E. Shladover, "Novel freeway traffic control with variable speed limit and coordinated ramp metering," Transportation Research Record, no. 2229, pp. 55-65, 2011.

[19] D. G. Hidalgo and M. Fontaine, "Impact of a variable speed limit system on driver speeds during low-visibility conditions," in Proceedings of the 97th Transportation Research Board Annual Meeting, (No. 18-03222), Washington, DC, USA, 2018.

[20] J. Fang, E. Hadiuzzaman, T. Qiu, M. Hadiuzzaman, and E. Yin, "DynaTAM: an online algorithm for performing simultaneously optimized proactive traffic control for freeways," Canadian Journal of Civil Engineering, vol. 41, no. 4, pp. 315-322, 2014.

[21] J. Fang, L. Tu, and S. M. Easa, "Ramp metering control using multi-objective optimization: mobility and safety," in Proceedings of the 97th Transportation Research Board Annual Meeting, (No. 18-01958), Washington, DC, USA, 2018. 


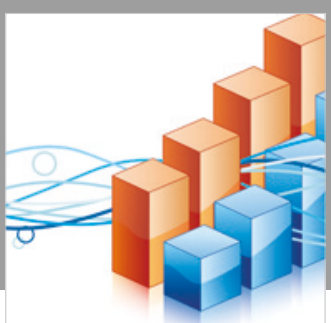

Advances in

Operations Research

\section{-n-m}
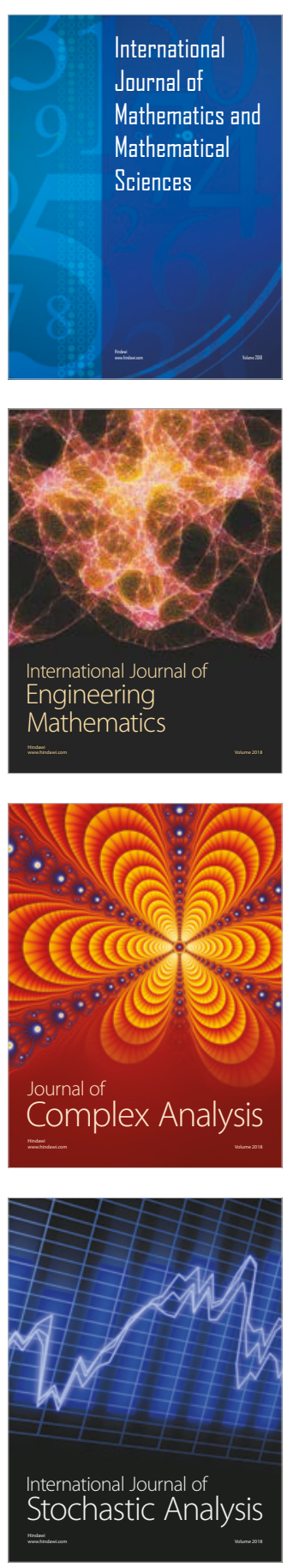
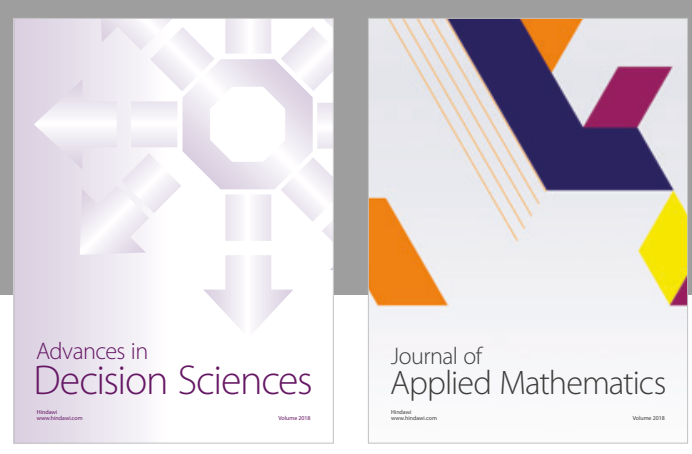

Journal of

Applied Mathematics
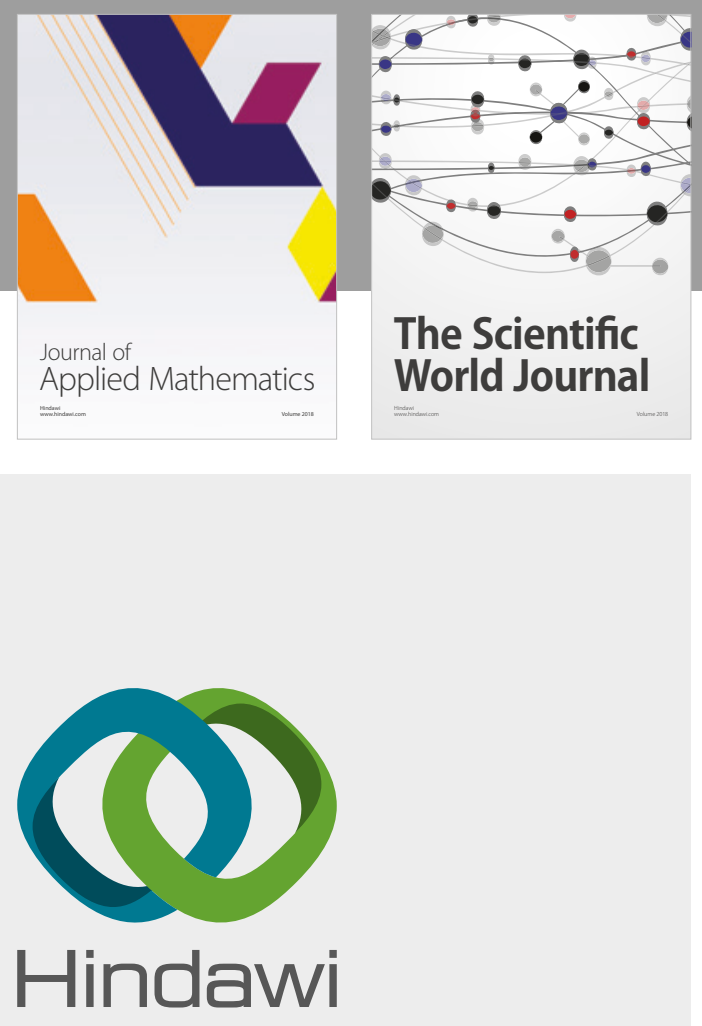

Submit your manuscripts at

www.hindawi.com

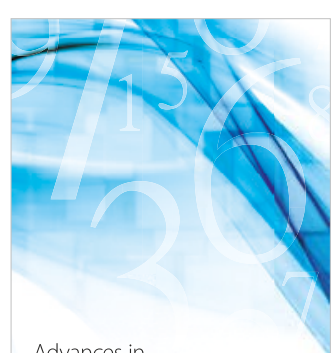

Advances in
Numerical Analysis
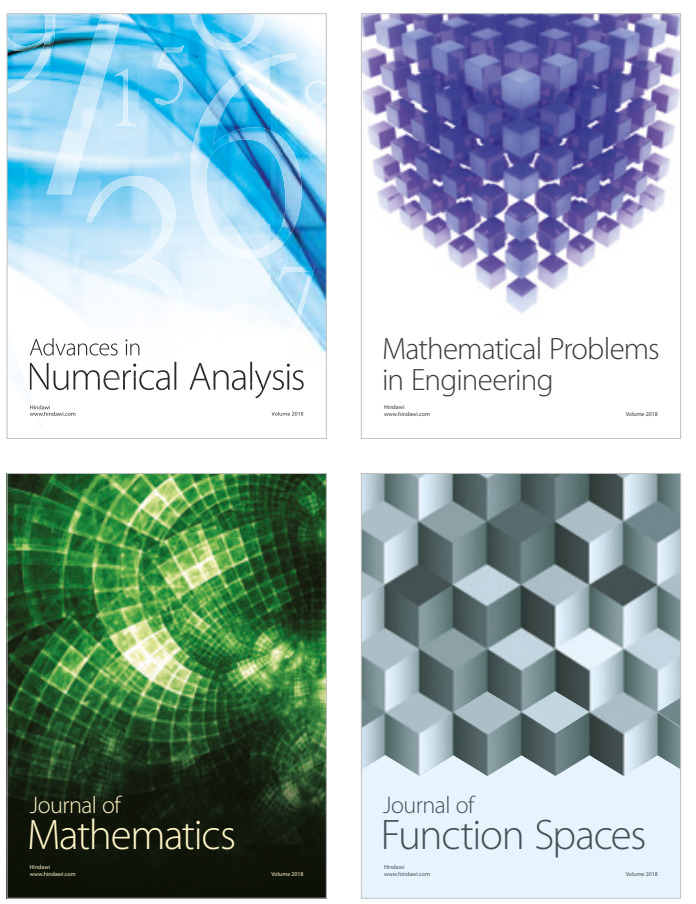

Mathematical Problems in Engineering

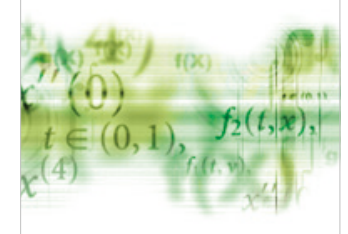

International Journal of

Differential Equations

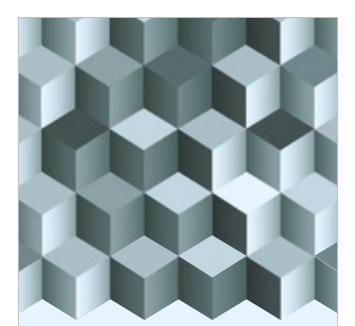

Journal of

Function Spaces

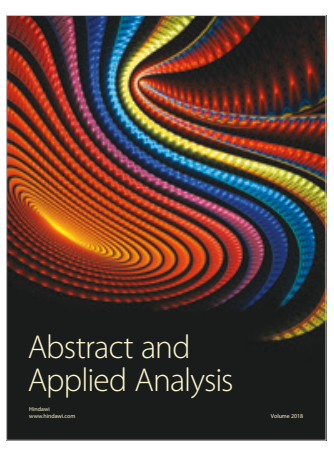

The Scientific

World Journal

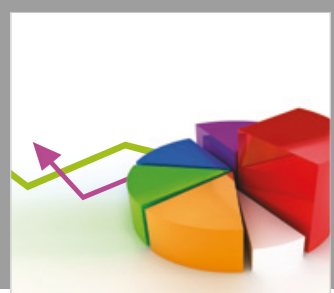

Journal of

Probability and Statistics
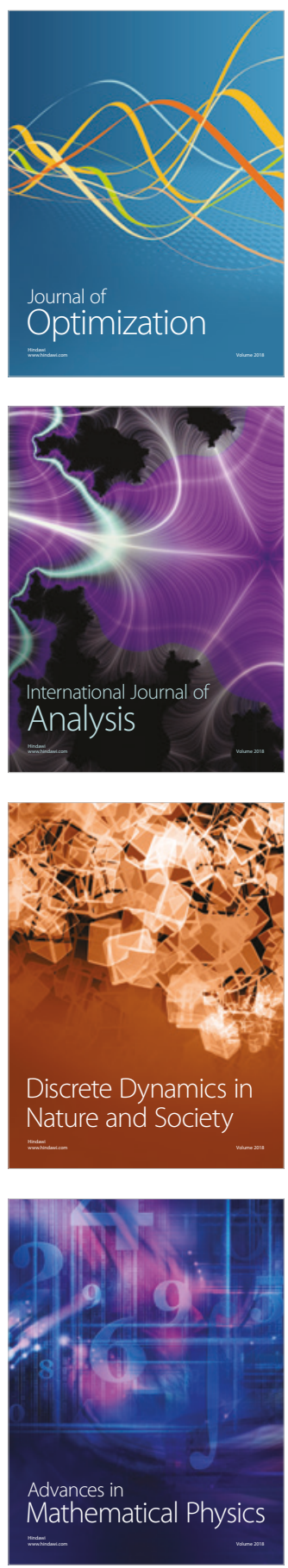